\title{
Incidência de lesão por pressão e tempo de assistência de enfermagem em terapia intensiva
}

\author{
Incidence of pressure injury and nursing care time in intensive care
Incidencia de lesiones por presión y tiempo de atención en enfermería en atención intensiva
Yasmin Cardoso Metwaly Mohamed Ali ${ }^{1, *}$, Taís Milena Pantaleão Souza ${ }^{2}$, Paulo Carlos Garcia ${ }^{3}$, Paula Cristina Nogueira ${ }^{1}$

ORCID IDS

Ali YCMM (iD https://orcid.org/0000-0001-9254-8062

Souza TMP iD https://orcid.org/0000-0003-2078-6103

Garcia PC (D) https://orcid.org/0000-0002-4591-1145

Nogueira PC (D) https://orcid.org/0000-0001-5200-1281
COMO CITAR

Ali YCMM; Souza TMP; Garcia PC; Nogueira PC. Incidência de lesão por pressão e tempo de assistência de enfermagem em terapia intensiva. ESTIMA, Braz. J. Enterostomal Ther., 18: e1120, 2020. https://doi. org/10.30886/estima.v18.849_PT

\begin{abstract}
RESUMO
Objetivos: Correlacionar a incidência de lesão por pressão (LP) com o tempo médio de assistência de enfermagem em unidade de terapia intensiva (UTI). Método: Estudo epidemiológico, observacional, retrospectivo, realizado em uma UTI de um hospital universitário. Os dados foram coletados pela consulta aos bancos de dados de incidência de LP e tempo médio de assistência de enfermagem entre 2010 e 2014. Utilizou-se medidas de tendência central e variabilidade, e coeficiente de correlação de Pearson para análise dos dados. Resultados: A média de incidência de LP entre 2010 e 2014 foi de 10,83\% (DP = 2,87) e o tempo médio de assistência de enfermagem despendido aos pacientes internados em UTI foi de 15 horas (DP = 0,94). Não houve correlação estatisticamente significante entre incidência de $L P$ e o tempo de assistência de enfermagem $(r=-0,17 ; p=0,199)$, porém os resultados sugeriram sobrecarga da equipe. Conclusão: Este estudo confirma a importância da implementação e reavaliação da eficácia de protocolos de cuidados preventivos para LP, além de alertar sobre a sobrecarga de trabalho de enfermagem na assistência aos pacientes críticos.
\end{abstract}

DESCRITORES: Incidência. Unidades de terapia intensiva. Cuidados de enfermagem. Lesão por pressão. Carga de trabalho. Estomaterapia.

\begin{abstract}
Objectives: To correlate the incidence of pressure injury (PI) with the average time of nursing care in an intensive care unit (ICU). Method: Epidemiological, observational, retrospective study, carried out in the ICU of a university hospital. Data were collected by consulting the PI incidence and the average nursing care time from ICU databases between 2010 and 2014. Measures of central tendency and variability, and Pearson's correlation coefficient were used for data analysis. Results: The average incidence of PI between 2010 and 2014 was 10.83\% (SD = 2.87) and the average time spent in nursing care for patients admitted to the ICU was 15 hours $(S D=0.94)$. There was no statistically significant correlation between the incidence of PI and the nursing care time $(r=-0.17$; $p=0.199)$, however, the results suggested an overload on the nursing team. Conclusion: This study confirms the importance of implementing and reassessing the effectiveness of preventive care protocols for PI, in addition to warning about the work overload of nursing in assisting critically ill patients.
\end{abstract}

DESCRIPTORS: Incidence. Intensive care units. Nursing care. Pressure ulcer. Workload. Enterostomal therapy.

\footnotetext{
1. Universidade de São Paulo - Escola de Enfermagem - Departamento de Enfermagem Médico-Cirúrgica - São Paulo (SP), Brasil. 2.Rede D'Or: Hospital Vila Nova Star - Unidade de Terapia Intensiva - São Paulo (SP), Brasil.

3. Universidade de São Paulo - Hospital Universitário - Unidade de Terapia Intensiva - São Paulo (SP), Brasil.
}

*Autor correspondente: yasminma16@gmail.com

Recebido: Mar. 2, 2020 | Aceito: Abr. 9, 2020 


\section{RESUMEN}

Objetivos: Correlacione la incidencia de úlceras por presión (UP) con el tiempo promedio de atención de enfermería en la Unidad de Cuidados Intensivos (UCI). Método: Estudio epidemiológico, observacional, retrospectivo, realizado en la UCI de un hospital universitario. Los datos se recopilaron consultando las bases de datos de incidencia de UP y el tiempo promedio de atención de enfermería entre 2010-2014. Se utilizaron medidas de tendencia central y variabilidad, y el coeficiente de correlación de Pearson para el análisis de datos. Resultados: La incidencia promedio de LP entre 2010-2014 fue de 10.83\% (DE = 2,87) y el tiempo promedio dedicado a la atención de enfermería para pacientes ingresados en la UCl fue de 15 horas (DE =0,94). No hubo correlación estadísticamente significativa entre la incidencia de UP y el tiempo de atención de enfermería ( $r=-0.17 ; p=0.199$ ), sin embargo, los resultados sugirieron una sobrecarga del equipo. Conclusión: Este estudio confirma la importancia de implementar y reevaluar la efectividad de los protocolos de atención preventiva para UP, además de advertir sobre la sobrecarga del trabajo de enfermería en la atención de pacientes críticos.

DESCRITORES: Incidencia. Unidades de cuidados intensivos. Atención de enfermería. Úlcera por presión. Carga de trabajo. Estomaterapia.

\section{INTRODUÇÃO}

A segurança do paciente é discutida mundialmente. Estudos acerca dessa temática destacam que um dos eventos adversos que mais afetam os pacientes hospitalizados é a lesão por pressão $(\mathrm{LP})^{1}$. As LPs causam dor e desconforto, aumentam o tempo de internação e a morbimortalidade, diminuem a qualidade de vida do paciente e de sua família, geram altos custos às instituições de saúde e aumentam a carga de trabalho da equipe de enfermagem. São considerados eventos adversos evitáveis, denominados “indicadores-chave", para mensurar a qualidade da assistência de enfermagem e o nível de segurança do paciente em ambiente hospitalar ${ }^{1-3}$.

O National Pressure Injury Advisory Panel(NPIAP) define as LPs como lesões que acometem a pele e/ou os tecidos subjacentes que se localizam geralmente sobre uma proeminência óssea, sendo causadas por pressão intensa ou ainda pela combinação de pressão e cisalhamento, que podem estar relacionadas ao uso de dispositivos médicos e outros artefatos ${ }^{4,5}$. O NPIAP classifica as LPs em quatro estágios (de 1 a 4); LP não classificável; LP tissular profunda; há ainda as LPs relacionadas a dispositivo médico, que são classificadas da mesma forma que as demais LPs; e as LPs em membranas mucosas que não são classificadas devido à anatomia do tecido ${ }^{4,5}$.

Existem vários fatores de risco relacionados ao paciente, ao processo de cuidar e à própria instituição que contribuem para o desenvolvimento das LPs. Pacientes críticos internados em unidades de terapia intensiva (UTI) são considerados mais vulneráveis e apresentam riscos ainda mais elevados para o desenvolvimento dessas lesões devido à imobilidade no leito, sedação, instabilidade clínica com necessidade do uso de drogas vasoativas e intervenções invasivas ${ }^{6}$.
As LPs são um dos eventos adversos mais prevalentes e incidentes na UTI, com taxas de incidência variando entre 8,8 a $25,1 \%$ no mundo todo ${ }^{3,6,7}$. Estudos nacionais revelam taxas de incidências entre 13,6 e 59,5\%2,8.

A incidência de LP reflete de forma indireta a qualidade do cuidado prestado, bem como é utilizada como parâmetro na avaliação de estratégias e elaboração de protocolos para prevenção. Dessa forma, a incidência de LP tem sido adotada como indicador de qualidade em serviço e da assistência de enfermagem por diversas instituições de saúde 3 .

Considerando os fatores de risco relacionados ao cuidado e às questões organizacionais, como o gerenciamento dos cuidados em saúde, que contribuem para eventos adversos, incluindo a LP, destacam-se a carga de trabalho e os recursos humanos em enfermagem. Apesar de existirem contestações sobre o quanto a carga de trabalho de enfermagem aumenta o risco de LP, vale ressaltar que pouco se tem discutido acerca desse tema na literatura ${ }^{3,9}$.

Estudos evidenciam a importância de considerar o coeficiente enfermeiro-paciente e o gerenciamento da carga de trabalho em enfermagem para integrar um conjunto de ações de prevenção de LP, proporcionando qualidade do cuidado e redução de custos das ações prestadas ${ }^{3}$. Dessa maneira, a capacitação da equipe de saúde quanto à identificação precoce dos fatores intrínsecos e extrínsecos é de suma importância no cuidado ao paciente crítico ${ }^{10,11}$.

Considerando a LP como um evento adverso que coloca em risco a segurança do paciente, quantificar e analisar a incidência de LP em UTI, assim como avaliar a correlação entre a incidência de LP e o tempo de assistência de enfermagem despendido a esses pacientes, faz-se relevante, pois colabora na avaliação da dimensão do problema e na 
elaboração de intervenções de enfermagem para prevenção de LP visando a qualidade da assistência, visto que a carga de trabalho de enfermagem no cuidado de LP tem sido pouco abordada na literatura, sobretudo a nacional.

Diante disso, este estudo objetivou identificar a incidência de LP e o tempo médio de assistência de enfermagem despendido aos pacientes internados na UTI e correlacionar a incidência de LP com o tempo médio de assistência de enfermagem despendido aos pacientes internados na UTI.

\section{MÉTODO}

Trata-se de um estudo epidemiológico, observacional, retrospectivo, de natureza quantitativa, realizado na UTI de um hospital universitário. A unidade possui o total de 12 leitos, sendo que quatro são destinados aos pacientes em isolamento. É um hospital geral, de ensino, localizado no município de São Paulo, considerado como serviço de referência de média complexidade e que integra o Sistema Único de Saúde (SUS).

A coleta de dados foi realizada por meio da consulta aos bancos de dados no programa Microsoft Excel, registrados pela chefia de enfermagem da UTI de acordo com as equações determinadas pelo Manual de Indicadores de Enfermagem $^{12}$, no período de janeiro de 2010 a dezembro de 2014, onde são armazenadas as informações referentes ao indicador de qualidade assistencial “incidência de LP”. Dados da incidência mensal de LPs são registrados nas planilhas do banco de dados. Também foram consultadas planilhas referentes ao tempo médio de assistência de enfermagem despendido aos pacientes internados na UTI.

Os dados foram coletados em 2016. Ao final de 2014 foram publicadas e divulgadas recomendações atualizadas para prevenção e tratamento de LP pela NPIAP, pela European Pressure Ulcer Advisory Panel (EPUAP) e pela Pan Pacific Pressure Injury Alliance (PPPIA) ${ }^{7}$. Com a publicação de novas diretrizes para prevenção e tratamento de LP, o protocolo de prevenção utilizado na instituição que foi construído com bases nas diretrizes da NPIAP e EPUAP de $2009^{13}$ iria passar por atualizações. Dessa forma, a escolha do período para coleta de dados foi de 2010 a 2014. Ressalta-se que foram realizados estudos na instituição sobre incidência de LPs em UTI até o ano de $2009^{14}$.

Os dados obtidos foram analisados de forma quantitativa empregando-se estatística descritiva, com medidas de tendência central e variabilidade através do software SPSS versão 20.0. A análise de correlação entre o indicador de qualidade assistencial "incidência de LP” e o tempo médio de assistência de enfermagem foi realizada por meio do coeficiente de correlação de Pearson e os resultados foram considerados estatisticamente significativos quando $\mathrm{p} \leq 0,05$.

Esta pesquisa foi aprovada pelo Comitê de Ética e Pesquisa (CEP) da instituição proponente pelo parecer $\mathrm{n}^{\circ}$ 1.235.310/CAAE 47336615.2.0000.5392 e pelo CEP do Hospital Universitário pelo parecer no 1.293.623/CAAE. 47336615.2.3001.0076.

\section{RESULTADOS}

Nas Tabelas 1 e 2 são apresentadas, respectivamente, os valores médios de incidência de LP e os valores médios do tempo de assistência de enfermagem, no período de 2010 a 2014.

Tabela 1. Valores médios de incidência mensal e anual de LP em UTI. São Paulo, 2020.

\begin{tabular}{|c|c|c|c|c|c|}
\hline Variáveis & 2010 & 2011 & 2012 & 2013 & 2014 \\
\hline Janeiro & 7,7 & 22,8 & 26,3 & - & 9,1 \\
\hline Fevereiro & 14,6 & 13,9 & 13,3 & 7,7 & 1,8 \\
\hline Março & 12,8 & 8,3 & 11,3 & 11,1 & 1,6 \\
\hline Abril & 8,5 & 12,8 & 17,1 & 12,5 & 15,8 \\
\hline Maio & 10,0 & 10,0 & 8,1 & 14,6 & 6,9 \\
\hline Junho & 17,3 & 7,7 & 10,6 & 14,9 & 2,5 \\
\hline Julho & 20,0 & 16,6 & 11,1 & 11,5 & 5,2 \\
\hline Agosto & 13,9 & 13,9 & 6,4 & 8,1 & 6,2 \\
\hline Setembro & 15,5 & 6,9 & 8,1 & 10,8 & 6,5 \\
\hline Outubro & 9,1 & 16,6 & 13,7 & 13,6 & 3,7 \\
\hline Novembro & 9,1 & 8,3 & 10,8 & 3,4 & 5,4 \\
\hline Dezembro & 9,4 & 3,3 & 15,4 & 19,1 & 3,9 \\
\hline Média anual & 12,3 & 11,8 & 12,7 & 11,6 & 5,7 \\
\hline Desvio padrão & 3,9 & 5,4 & 5,3 & 4,2 & 3,9 \\
\hline Mínimo & 7,7 & 3,3 & 6,4 & 3,4 & 1,6 \\
\hline Máximo & 20,0 & 22,8 & 26,3 & 19,1 & 15,8 \\
\hline
\end{tabular}

A média de incidência de LP considerando o período de 2010 a 2014 foi de 10,83\% (DP = 2,87), sendo a menor média de incidência 5,7\% (DP $=3,9)$ no ano de 2014 e a maior média de incidência $12,7 \%(\mathrm{DP}=5,3)$ no ano de 2012. Destaca-se que, pontualmente em janeiro de 2012, a 
maior média de incidência de LP chegou a 26,3\%, enquanto a menor média (1,6\%) ocorreu em março de 2014.

Tabela 2. Valores médios do tempo de assistência de enfermagem em uma unidade de terapia intensiva adulta, segundo mês e ano. São Paulo, 2020.

\begin{tabular}{cccccc}
\hline Variáveis & $\mathbf{2 0 1 0}$ & $\mathbf{2 0 1 1}$ & $\mathbf{2 0 1 2}$ & $\mathbf{2 0 1 3}$ & $\mathbf{2 0 1 4}$ \\
Janeiro & 13,5 & 14,0 & 14,9 & 13,6 & 14,8 \\
\hline Fevereiro & 13,0 & 15,2 & 15,9 & 15,0 & 15,6 \\
\hline Março & 13,7 & 16,0 & 15,5 & 16,9 & 16,0 \\
\hline Abril & 13,0 & 15,6 & 14,9 & 14,5 & 15,2 \\
\hline Maio & 14,0 & 16,3 & 13,8 & 14,5 & 14,0 \\
\hline Junho & 13,6 & 15,7 & 15,0 & 15,8 & 15,2 \\
\hline Julho & 13,2 & 15,8 & 15,8 & 14,6 & 15,9 \\
\hline Agosto & 14,1 & 14,2 & 15,6 & 13,7 & 17,9 \\
\hline Setembro & 14,1 & 14,7 & 14,7 & 14,0 & 14,3 \\
\hline Outubro & 14,7 & 17,1 & 16,7 & 16,5 & 15,7 \\
\hline Novembro & 15,2 & 14,2 & 16,0 & 16,7 & 13,4 \\
\hline Dezembro & 14,5 & 15,4 & 16,4 & 15,1 & 16,5 \\
\hline Média & 13,8 & 15,3 & 15,4 & 15,1 & 15,4 \\
\hline Desvio Padrão & 0,7 & 0,9 & 0,8 & 1,1 & 1,2 \\
\hline Mínimo & 13 & 14 & 13,8 & 13,6 & 13,4 \\
\hline Máximo & 15,2 & 17 & 16,7 & 16,9 & 17,9 \\
\hline
\end{tabular}

O tempo médio de assistência de enfermagem despendido aos pacientes internados em UTI foi de 15 horas (DP $=0,94)$, sendo observada a menor média nos meses de fevereiro e abril de 2010 (13 horas) e a maior média (17,9 horas) em agosto de 2014.

Ao correlacionar a incidência de LP com o tempo de assistência de enfermagem, obteve-se uma correlação negativa e fraca $(r=-0,17 ; p=0,199)$. Uma correlação negativa demonstra uma linearidade negativa entre as variáveis, ou seja, permite inferir que a incidência de LP diminui à medida que aumenta o tempo de assistência de enfermagem (Fig. 1). Porém valores de "r" até 0,30 são considerados correlação fraca e de pouca aplicabilidade clínica, mesmo quando estatisticamente significantes.

\section{DISCUSSÃO}

Evidências científicas apontam altos índices de incidência de LP em pacientes hospitalizados, especialmente em UTI, devido aos inúmeros fatores de risco aos quais estão $\operatorname{expostos}^{3,10}$.No cenário mundial, estudo de revisão sistemática e metanálise identificou uma incidência cumulativa de 10,0 a 25,9\% (IC 95\%). Nos estudos dessa revisão em que a inspeção cutânea foi realizada para identificar as LP, a incidência acumulada foi de 9,4 a 27,5\% (IC 95\%) e nos estudos que excluíram o estágio 1 , a incidência foi de 0,0 a 23,8\% (IC 95\%) $)^{15}$.

Em estudo desenvolvido com 335 pacientes internados em UTIs de hospitais da Espanha, acompanhados por no máximo 32 dias, foi identificada uma taxa de incidência de 8,1\% de LP ${ }^{17}$. Na Arábia Saudita, 84 pacientes internados em UTIs foram avaliados no período de 30 dias e encontrou-se 39,3\% de incidência de LP ${ }^{1}$. Estudo retrospectivo desenvolvido em dois hospitais do Irã, com uma amostra de 643 pacientes, a incidência de LP foi de 8,9\% ${ }^{6}$.

Considerando a incidência de LP descrita em trabalhos científicos nacionais, estudo multicêntrico e prospectivo desenvolvido em um hospital universitário do Paraná, que incluiu 10 UTIs gerais e avaliou 332 pacientes admitidos durante 31 dias, encontrou uma incidência de $13,6 \%{ }^{8}$. Outro estudo desenvolvido com 77 pacientes internados em UTI

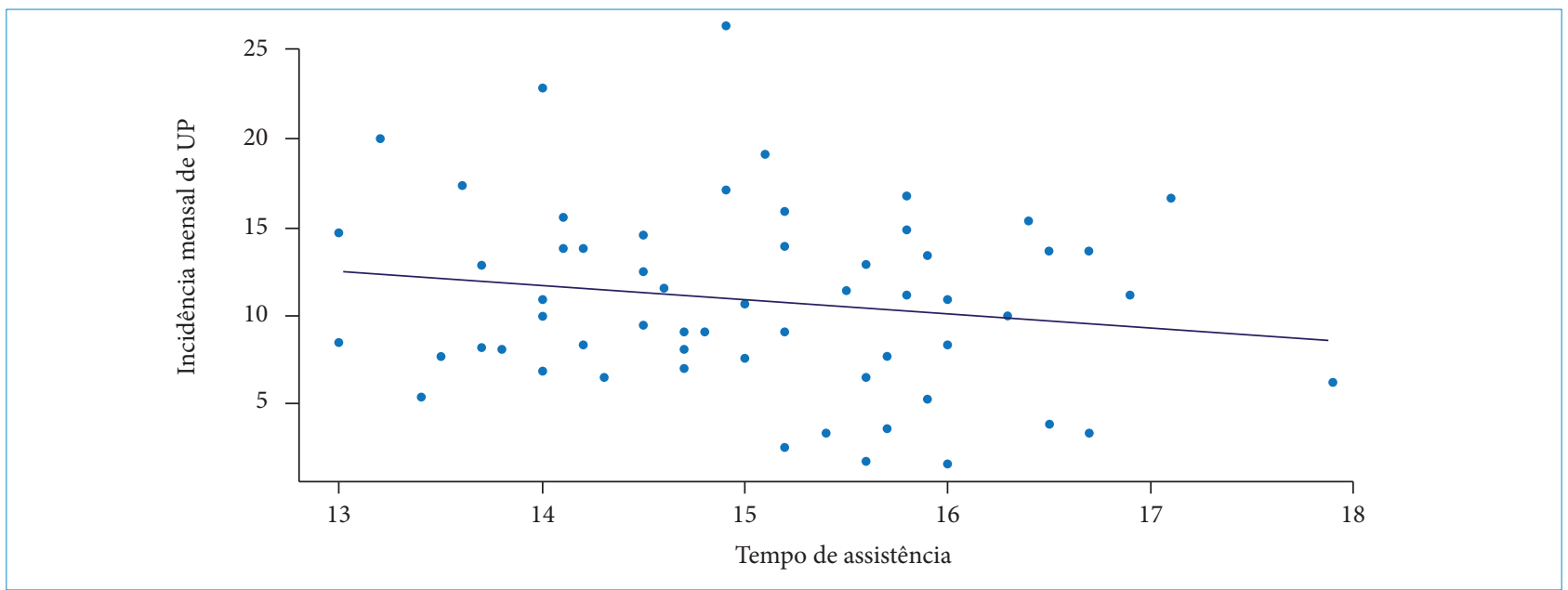

Figura 1. Dispersão da incidência de lesão por pressão pelo tempo de assistência de enfermagem. São Paulo, 2020. 
do Hospital Universitário de Vitória/ES, avaliados no período de quatro meses, identificou que a incidência de LP foi de $22 \%{ }^{2}$. Estudo de coorte retrospectiva realizado com 766 pacientes, de nove UTIs de dois hospitais universitários localizados na cidade de São Paulo, identificou uma incidência de LP de $18,7 \%{ }^{3}$.

Os dados obtidos no presente estudo expressaram que a média anual de incidência de LP, considerando o período de 2010 a 2014, foi de 10,83\%, sendo que o ano de 2014 apresentou a menor média de incidência anual de LP $(5,7 \%$, $\mathrm{DP}=3,9)$ e a maior média anual $(12,7 \%, \mathrm{DP}=5,3)$ foi observada no ano de 2012. Ao considerar a incidência mensal, as maiores médias, com valores acima de $20 \%$, ocorreram em janeiro de 2011, quando foi identificada incidência de LP de 22,8\% e em janeiro de 2012 com 26,3\%.

Em 2005, foi realizado o primeiro estudo que investigou a incidência de LP em diversas unidades, durante o período de três meses consecutivos, no mesmo hospital onde foi realizado o presente estudo. A partir dessa investigação, foi identificada a incidência de $41 \%$ de LP na UTI ${ }^{18}$. Com base nesse dado, um protocolo de prevenção de LP foi implementado na instituição pelo Grupo de Estudo de Enfermagem em Estomaterapia. O protocolo de prevenção foi construído com base nas diretrizes do NPIAP, EPUAP e PPPIA de $2009^{13}$. Além disso, a incidência de LP passou a ser considerada um indicador de qualidade na instituição ${ }^{12}$.

Em 2012, foi realizado o segundo estudo que avaliou a incidência de LP após a implementação do protocolo de prevenção e a incidência identificada na UTI foi de $23,1 \%$, destacando importante diminuição na incidência de LP, evidenciando que o protocolo implementado impactou positivamente a prevenção de novos $\operatorname{casos}^{14}$. No presente estudo constatou-se que a incidência de LP foi diminuindo gradativamente com o passar dos anos, sendo o valor máximo $26,3 \%$, observado pontualmente em janeiro de 2012. Dessa forma, nota-se a necessidade de maior atenção para prevenção de novos casos para que a incidência permaneça diminuindo por meio do suporte da administração da instituição, no fornecimento dos recursos materiais e humanos, e no ativo envolvimento dos profissionais ${ }^{3,19-21}$.

Quanto ao tempo de assistência de enfermagem ao paciente na instituição, a média entre os anos de 2001 a 2005 foi de 15,4 horas/dia ${ }^{22}$. Entre 2008 e 2009, a média de tempo foi 14 horas $/ \mathrm{dia}^{23}$. A média entre os anos de 2010 a 2014 foi de 15 horas/dia (DP = 0,94), variando de 13,8 a 15,4 horas/dia, sendo observada a menor média com 13 horas em fevereiro e abril de 2010, e a maior média com 17,9 horas em agosto de 2014.

Com relação aos valores estabelecidos pelo Conselho Federal de Enfermagem (Cofen), de acordo com a Resolução Cofen no 543/2017, o tempo médio de assistência de enfermagem a pacientes internados em UTI é de 18 horas $^{24}$. Portanto observa-se que a média de horas de assistência de enfermagem despendidas aos pacientes internados na UTI do hospital universitário em questão, em todos os meses do período de 2010 a 2014, foram inferiores às indicadas pelo $\mathrm{Cofen}^{24}$, o que pode sugerir sobrecarga de trabalho da equipe de enfermagem.

Com resultados diferentes ao encontrado no presente estudo, pesquisa realizada em 11 UTIs de três hospitais localizados na cidade de São Paulo, sendo dois hospitais públicos e um hospital privado, identificou um tempo médio de assistência de enfermagem superior aos valores preconizados pelo Cofen, sendo 18,86 horas (hospital público A), 21 horas (hospital público B) e 19,50 horas (hospital privado) ${ }^{25}$.

Ao analisar a correlação entre a incidência de LP e o tempo de assistência de enfermagem, não foi encontrada significância estatística, ou seja, o tempo de assistência não pode ser considerado determinante para que o paciente apresente LP,já que se apresentam inversamente proporcionais.

Em outro estudo realizado na mesma UTI, em 2011, com o objetivo de analisar o tempo de assistência da equipe de enfermagem e verificar sua correlação com os indicadores de qualidade assistencial, evidenciou-se que houve correlação estatisticamente significante entre o tempo de assistência de enfermagem e o indicador de incidência de extubação acidental, que diminuiu à medida que o tempo de assistência de enfermagem despendido por enfermeiros aumentou. Com relação ao indicador de enfermagem "incidência de LP", os autores também identificaram uma correlação negativa, fraca e sem significância estatística $(r=-0,162 ; p=0,450)^{23}$.

Estudos brasileiros avaliaram o tempo de assistência de enfermagem a partir do nursing activities score (NAS); um deles, desenvolvido no ano de 2012 com 766 pacientes em nove UTIs de dois hospitais universitários, encontrou que a razão de possibilidades (RP) do desenvolvimento de LP aumentou 1,5\% para cada ponto registrado no NAS. Dessa forma, a carga de trabalho de enfermagem foi identificada como um preditor de $\mathrm{LP}^{3}$. Já o outro estudo realizado em 3 UTIs de um hospital universitário de grande porte localizado no município de São Paulo, no período de novembro de 
2007 a abril de 2008, identificou que o NAS atuou como um fator de proteção, porque sua razão de possibilidades foi $<1(\mathrm{RP}=0,916$; IC95\% = 0,855-0,98). Assim, pacientes com alta carga de trabalho de enfermagem tinham menos chance de desenvolver LP ${ }^{16}$.

O paciente crítico apresenta diversos fatores de risco associados, sejam individuais, clínicos e terapêuticos, que o predispõem ao desenvolvimento de LP ${ }^{10,15}$. Neste estudo, o tempo de assistência despendido ao paciente crítico não foi considerado determinante para o desenvolvimento de LP. No entanto, os diferentes valores encontrados na literatura, acerca do tempo de assistência de enfermagem, podem ser explicados pelas características dos pacientes, das UTI e dos hospitais. Além disso, esse indicador possui maior impacto em conjunto com demais fatores como: faixa etária, estado nutricional, longos períodos submetidos à umidade, ventilação mecânica, uso de drogas vasoativas, instabilidade hemodinâmica e restrição de movimentos, para que o surgimento de LP seja mais preditivo ${ }^{10}$. Ressalta-se que tais fatores não foram avaliados no presente estudo.

\section{CONCLUSÃO}

Os resultados deste estudo identificaram diminuição da incidência de LP em comparação com os estudos anteriores realizados na mesma instituição, o que pode ser atribuído à eficácia da implementação do protocolo de prevenção na UTI e à efetividade das contínuas adequações de tais medidas, tornando os casos de LP cada vez menores. Não houve significância estatística na correlação entre incidência de LP e o tempo de assistência de enfermagem. No entanto os resultados sugeriram sobrecarga da equipe de enfermagem, pois as horas médias de assistência de enfermagem despendidas aos pacientes críticos em todos os meses, do período de 2010 a 2014, foram inferiores às indicadas pelo Cofen (18 horas).

Os resultados deste trabalho demonstram que novos estudos devem ser realizados, pois há escassez de literatura acerca da carga de trabalho da enfermagem e a ocorrência de LP em suas diversas classificações. Vale ressaltar que este estudo utilizou dados retrospectivos de apenas uma UTI com doze leitos; assim, estudos prospectivos com amostras maiores, envolvendo outros hospitais e UTIs e com tempo de seguimento maior fazem-se necessários para reforçar a evidência da relação entre carga de trabalho de enfermagem e desenvolvimento de LP.

Por fim, o presente estudo confirma a importância da implementação de protocolos de cuidados preventivos para LP e reavaliação da eficácia desses protocolos. Além disso, alerta sobre a sobrecarga de trabalho de enfermagem, visando uma assistência segura e qualificada ao paciente crítico.

\section{AGRADECIMENTOS}

À Unidade de Terapia Intensiva do Hospital Universitário da Universidade de São Paulo.

\section{CONTRIBUIÇÃO DOS AUTORES}

Conceitualização, Nogueira PC e Ali YCMM; Metodologia, Nogueira PC, Garcia PC e Ali YCMM; Redação - Revisão \& Edição, Souza TMP, Ali YCMM e Nogueira PC; Supervisão, Nogueira PC e Ali YCMM.

\section{REFERÊNCIAS}

1. Tayyib N, Coyer FM, Lewis P. Saudi Arabian adult intensive care unit pressure ulcer incidence and risk factors: a prospective cohort study. Int Wound J. 2016;13(5):912-9. https://doi.org/10.1111/iwj.12406

2. Borghardt AT, Prado TN, Bicudo SDS, Castro DS, Bringuente MEO. Pressure ulcers in critically ill patients: incidence and associated factors. Rev Bras Enferm. 2016;69(3):431-8. https://doi.org/10.1590/0034-7167.2016690307i

3. Strazzieri-Pulido KC, González CVS, Nogueira PC, Padilha $K G$, Santos VLCG. Pressure injuries in critical patients: incidence, patient-associated factors, and nursing workload. J Nurs Manag. 2019;27(2):301-10. https://doi.org/10.1111/ jonm.12671

4. European Pressure Ulcer Advisory Panel. National Pressure Injury Advisory Panel. Pan Pacific Pressure Injury Alliance. Prevention and treatment of pressure ulcers: quick reference guide [Internet]. Emily Haesler, editor. Prevention and Treatment of Pressure Ulcers/Injuries: Quick Reference Guide. 2019. [citado em 24 Mar 2020] Disponível em: https://www.epuap.org/download/11182/ 
5. Associação Brasileira de Estomaterapia (SOBEST), Associação Brasileira de Enfermagem em Dermatologia (SOBENDE). Classificação das lesões por pressão - consenso NPUAP 2016 - adaptada culturalmente para o Brasil. [citado em 24 Mar 2020]. Disponível em: http://www.sobest.org. $\mathrm{br} /$ textod/35

6. Zarei E, Madarshahian E, Nikkhah A, Khodakarim S. Incidence of pressure ulcers in intensive care units and direct costs of treatment: evidence from Iran. J Tissue Viability. 2019;28(2):70-4. https://doi.org/10.1016/j.jtv.2019.02.001

7. National Pressure Ulcer Advisory Panel (NPUAP), European Pressure Ulcer Advisory Panel (EPUAP), Pan Pacific Pressure Injury Advisory (PPPIA). Prevenção e tratamento de úlceras por pressão: guia de consulta rápida. Osborne Park: Cambridge Media; 2014. [citado em 24 Mar 2020]. Disponível em: https://www.epuap.org/wp-content/ uploads/2016/10/portuguese-quick-reference-guidejan2016.pdf

8. Becker D, Tozo TC, Batista SS, Mattos AL, Silva MCB, Rigon $\mathrm{S}$, et al. Pressure ulcers in ICU patients: Incidence and clinical and epidemiological features: a multicenter study in southern Brazil. Intens Crit Care Nur. 2017;42:55-61. https://doi.org/10.1016/j.iccn.2017.03.009

9. Oliveira AC, Garcia PC, Nogueira LS. Nursing workload and occurrence of adverse events in intensive care: a systematic review. Rev Esc Enferm USP. 2016;50(4):679-89. https://doi. org/10.1590/S0080-623420160000500020

10. Campanili TCGF, Santos VLCG, Strazzieri-Pulido KC, Thomaz PBM, Nogueira PC. Incidência de úlceras por pressão em pacientes de unidade de terapia intensiva cardiopneumológica. Rev Esc Enferm USP. 2015;49(Esp):714. https://doi.org/10.1590/S0080-623420150000700002

11. Lainseck FGT, Rodrigues LHN, Ramos TM, Silva KCC, Muniz CF, Barcellos VM. Incidência de úlceras de pressão no hospital regional de Gurupi-TO. Rev Cereus. 2016;8(1):182-99. https:// doi.org/10.18605/2175-7275/cereus.v8n1 p182-199

12. Compromisso com a qualidade hospitalar (CQH). Manual de indicadores de enfermagem NAGEH/Compromisso com a qualidade hospitalar (CQH). São Paulo: APM/CREMESP; 2012. [citado em 24 Mar 2020]. Disponível em: http://www. cqh.org.br/portal/pag/doc.php?p_ndoc=125

13. National Pressure Ulcer Advisory Panel (NPUAP), European Pressure Ulcer Advisory Panel (EPUAP). Treatment of pressure ulcers: quick reference guide. Washington: National Pressure Ulcer Advisory Panel; 2009. [citado em 24 Mar 2020]. Disponível em: https://gneaupp.info/wpcontent/uploads/2014/12/pressure-ulcer-treatment.pdf

14. Rogenski NMB, Kurcgant P. The incidence of pressure ulcers after the implementation of a prevention protocol.
Rev Latino-Am Enfermagem. 2012;20(2):333-9. https://doi. org/10.1590/S0104-11692012000200016

15. Chaboyer WP, Thalib L, Harbeck EL, Coyer FM, Blot S, Bull $\mathrm{CF}_{\text {, et }}$ al. Incidence and prevalence of pressure injuries in adult intensive care patients: a systematic review and metaanalysis. Crit Care Med. 2018;46(11):e1074-81. https://doi. org/10.1097/CCM.0000000000003366

16. Cremasco MF, Wenzel F, Zanei SSV, Whitaker IY. Pressure ulcers in the intensive care unit: the relationship between nursing workload, illness severity and pressure ulcer risk. J Clin Nurs. 2013;22(15-16):2183-91. https://doi. org/10.1111/j.1365-2702.2012.04216.x

17. González-Méndez MI, Lima-Serrano M, Martín-Castaño C, Alonso-Araujo I, Lima-Rodríguez JS. Incidence and risk factors associated with the development of pressure ulcers in an intensive care unit. J Clin Nurs. 2018;27(5-6):1028-37. https://doi.org/10.1111/jocn.14091

18. Rogenski NMB, Santos VLCG. Estudo sobre a incidência de úlceras por pressão em um hospital universitário. Rev Latino-Am Enfermagem. 2005;13(4):474-80. https://doi. org/10.1590/S0104-11692005000400003

19. Farías IA, Febré N. Impacto de un programa de gestión de riesgo en la tasa de úlceras por presión. Rev Calid Asist. 2017;32(6):322-7. https://doi.org/10.1016/j. cali.2017.10.003

20. Ministério da Saúde (Brasil). Portaria n. 1.377, de 9 de julho de 2013. [citado em 24 Mar 2020]. Disponível em: http://bvsms.saude.gov.br/bvs/saudelegis/gm/2013/ prt1377_09_07_2013.html

21. Vasconcelos JMB, Caliri MHL. Ações de enfermagem antes e após um protocolo de prevenção de lesões por pressão em terapia intensiva. Esc Anna Nery. 2017; 21(1):e20170001.

22. Rogenski KE, Fugulin FMT, Gaidzinski RR, Rogenski NMB. Tempo de assistência de enfermagem em instituição hospitalar de ensino. Rev Esc Enferm USP. 2011;45(1):2239. https://doi.org/10.1590/S0080-62342011000100031

23. Garcia PC, Fugulin FMT. Nursing care time and quality indicators for adult intensive care: correlation analysis. Rev Latino-Am Enfermagem. 2012; 20(4):1-9. https://doi. org/10.1590/S0104-11692012000400004

24. Conselho Federal de Enfermagem (Brasil). Resolução n 543/2017, de 12 de maio de 2017. [citado em 24 Mar 2020]. Disponível em: http://www.cofen.gov.br/resolucaocofen-5432017_51440.html

25. Garcia PC, Tronchin DMR, Fugulin FMT. Care time and quality indicators in Intensive Care Units. Rev Bras Enferm. 2019;72(1 Suppl):166-72. https://doi.org/10.1590/00347167-2018-0067 